\title{
A Broadly Protecting Influenza Vaccine Relies on New Adjuvans Effects
}

\author{
BrorMorein* \\ Department of Biomedical Sciences and Veterinary Public Health, Swedish University of Agricultural Sciences,Sweden
}

Submission: May 20, 2018; Published: July 02, 2018

*Corresponding author: BrorMorein,Department of Biomedical Sciences and Veterinary Public Health, Swedish University of Agricultural Sciences,Sweden, Tel: +46705521859; Email: bror.morein@gmail.com

Keywords: Vaccine;Immunogenic; Pathogens; Virus; Mortality; Morbidity; Viroscience; Nanoparticle; polymerase; Glycoside;Humans; Antibodies; Infections; Antigen determinants; Adjuvants

\section{Short Communication}

Vaccines used against seasonal influenza are poorly effective against influenza A viruses of novel subtypes that may cause pandemic. Pre-pandemic influenza vaccines are poorly immunogenic against the upcoming pandemic virus that might be overcome by the use of a potent adjuvant. In humans, a limited number of adjuvants has been approved for human use and mostly human influenza vaccines are not adjuvanted. Furthermore, pre-pandemic influenza vaccines are poorly immunogenic to the upcoming pandemic. Thus, there is a need for alternative safe and effective adjuvants that can enhance the immunogenicity of influenza vaccines and that promote the induction of broad-protective $\mathrm{T}$ cell responses. Most studies of pandemic outbreaks have been done in humans. Also in animal spp problems exist with influenza virus infections. However, in the animal field the situation is different where one and the same influenza virus strain may circulate in the population finding new accessible victims in new non-exposed individuals.

Influenza virus infections cause severe morbidity and mortality in the human population [1]. For overcoming such problems annual vaccination of high risk groups is recommended. The inactivated trivalent influenza vaccines are used that elicit strain-specific antibodies [2,3]. However, these vaccines fail to afford long lasting protection due to antigenic drift of influenza viruses [4-6] but also human Influenza vaccines are not highly immunogenic requiring regular updating.

Vaccine strains not matching the infecting virus have reduced effectiveness [7]. The emergence of an antigenically distinct influenza virus of a novel subtype triggers a pandemic to which inactivated seasonal influenza vaccines afford little protection [4]. Thus, it is obvious that influenza virusvaccine should be formulated that not only induce neutralizing antibodies to seasonal influenza viruses but also induce more broadly protective immune responses over alternative protective antigenic determinants also on alternative proteins and recruiting alternative immune mechanisms.

In a recent study at Carolien E,et al. [6], Dept of viroscience, Erasmus university MC,) Rotterdam, The Netherlands evaluated a novel nanoparticle, G3, as an adjuvant in a seasonal trivalent inactivated influenza vaccine in a mouse model. The G3 adjuvant was formulated with (G3/DT: DT, for diterpenoid). or without steviol glycoside diterpenoid. The use of both formulations enhanced potently the virus-specific antibody responses to all three vaccine strains. The adjuvants were well tolerated without any signs of discomfort. To assess the protective potential of the vaccine-induced immune responses, an antigenically distinct H1N1 influenza virus strain, A/Puerto Rico/8/34 (A/PR/8/34), was used for challenge infection. This strain was selected because it like the H1N1 virus in the most recent influenza virus outbreak lacked hemagglutination (HI) and virus neutralizing (VN) epitopes on the HA-protein.The vaccine-induced antibodies did not recognize $\mathrm{HI}$ or $\mathrm{VN}$ antigenic determinants on the challenge virus i.e. it did not cross-react with strain $\mathrm{A} / \mathrm{PR} / 8 / 34$ in $\mathrm{HI}$ and VN assays. However, mice immunized with the G3/DT-adjuvanted vaccine showed Protective immunity against A/PR/8/34 infection, which correlated with the induction of anamnestic virus-specific CD8+ T cell responses to epiotopes located on the structural nukleo protein (NP) and more remarkable on the nonstructural polymerase A (PA), which were not observed with the use of G3 without DT.

G3 and G3/DT enhance the vaccine-induced antibody responses upon vaccination none of the mice displayed any signs of discomfort. Limited weight loss $(<2 \%)$ was observed during 
two days post vaccination in some mice randomly distributed over the experimental groups, including those that received PBS only All mice that received the trivalent inactivated influenza vaccine i.e. non-adjuvanted vaccine or vaccine adjuvanted with G3 alternatively with G3/DT developed HI antibody titres against the three vaccine strains (A/California/7/2009, A/ Victoria/361/2011 and B/Hubei-Wujiang/158/2009) after the first vaccination. The geometric mean titers (GMTs) of mice that received the vaccine with adjuvant G3/DT or G3 were significantly higher than those of mice that received the nonadjuvanted vaccine.Four weeks after the second vaccination the GMTs against the vaccine strains increased in the mice receiving the non-adjuvanted vaccine. Although the GMTs of groups that received the G3/DT and the G3 adjuvanted vaccine respectively, did not increase after the second vaccination, they remained significantly higher than the GMTs of receiving non-adjuvanted vaccine adjuvant) $(\mathrm{p}<0.01)$.

In none of the mice, vaccine-induced antibodies were cross-reactive with the influenza virus challenge strain $\mathrm{A} /$ $\mathrm{PR} / 8 / 34$. Virus-specific CD8+ T cells were detected in the G3/ DT adjuvanted vaccine group only. The CD8+ T cells were specific for the NP366-374 and the PA224-233 epitopes present in the A/ $\mathrm{PR} / 8 / 34$ influenza $A$ vaccine strains and the $\mathrm{A} / \mathrm{PR} / 8 / 34$ virus used for challenge infection. Thus, CD8+ T cells had contributed to the protection observed in mice that received the G3/DTadjuvanted vaccine. Weight loss is a disease criterion and all mice vaccinated or not displayed similar weight loss, starting at day 2 p.i. until day 4 p.i. From day 5 p.i. onwards mice vaccinated with the G3/DT-adjuvanted vaccine started to gain weight and recovery. Furthermore, The G3/DT adjuvanted vaccine restricted virus replication in the lungs compared to the other two vacc8ine groups van De Sandt[7]

\section{Conclusion}

G3/DT improves the protective efficacy of the split virion vaccine against an antigenically distinct influenza virus challenged two weeks after the second vaccination mice with a lethal dose of influenza A/PR/8/34.

Regardless the influenza virus infects man or animal there is a need for update of Influenza virus vaccines. Here we give clear evidence that influenza virus vaccine can be formulated to inducing broadened immune protection. Most important now is to define \& design efficient adjuvants recruiting new antigen and antigen determinants and alternative immune mechanisms. Here, I like to underline an innovative mechanism of the G3/DT adjuvant that can enhance the immune mechanism harboured in RNA by promoting expression of the non-structural PA containing an immune protective epitope. That and other effects named in this article should stimulate adjuvant development and open for enhancing protection mechanisms overcoming the evasion mechanisms of influenza virus as well of other pathogens.

\section{References}

1. WHO (2014) Influenza (seasonal) WHO, Geneva, Switzerland.

2. Cox RJ, Brokstad KA, Ogra P (2004) Influenza virus: immunity and vaccination strain. Comparison of the immune response to inactivated and live, attenuated influenza vaccines. Scand J Immunol 59(1): 1-15.

3. De Jong JC, Rimmelzwaan GF, Fouchier RA, Osterhaus AD (2000) Influenza virus: a master of metamorphosis. J Infect 40(3): 218-228.

4. Rambaut A, Pybus OG, Nelson MI, Viboud C, Taubenberger JK (2008) The genomic and epidemiological dynamics of human influenza $\mathrm{A}$ virus. Nature 453(7195): 615-619.

5. Smith DJ, Lapedes AS, de Jong JC, Bestebroer TM, Rimmelzwaan GF et al. (2004) Mapping the antigenic and genetic evolution of influenza virus. Science 305(5682): 371-376.

6. Carolien E, van de Sandta, Joost HCM, Kreijtza Martina M, GeelhoedMierasa et al. \# NovelG3/DT adjuvant promotes the induction of protective $\mathrm{T}$ cells responses after vaccination with a seasonal trivalent inactivated split-virion influenza vaccine

7. de Jong JC, Beyer WE, Palache AM, Rimmelzwaan GF, Osterhaus AD (2000) Mismatch between the 1997/1998 influenza vaccine and the major epidemic $\mathrm{A}(\mathrm{H} 3 \mathrm{~N} 2)$ virus strain as the cause of an inadequate vaccine-induced antibody response to this strain in the elderly. J Med Virol 61(1): 94-99.

\section{Your next submission with Juniper Publishers will reach you the below assets}

- Quality Editorial service

- Swift Peer Review

- Reprints availability

- E-prints Service

- Manuscript Podcast for convenient understanding

- Global attainment for your research

- Manuscript accessibility in different formats

( Pdf, E-pub, Full Text, Audio)

- Unceasing customer service

Track the below URL for one-step submission

https://juniperpublishers.com/online-submission.php 